\title{
Effective Image Retrieval Supporting Complex Similarity Queries
}

G.-H. Cha ${ }^{1}$ and C.-W. Chung ${ }^{2}$

${ }^{1}$ Department of Multimedia Engineering

Tongmyong University of Information Technology

Pusan 608-080, South Korea,

Tel: +82-51-629-7269, Fax: +82-51-629-7249,

E-mail: ghcha@tmic.tit.ac.kr

${ }^{2}$ Department of Computer Science,

Korea Advanced Institute of Science and Technology

Taejon 305-701, South Korea,

Tel: +82-2-958-3316, Fax: +82-2-958-3384,

E-mail: chungcw@ngis.kaist.ac.kr

\begin{abstract}
A content-based image retrieval system to support complex similarity queries consisting of more than one similarity query is presented. The indexing scheme and evaluation strategies for complex similarity queries are elaborated. In the system, three types of indexes exist: visual indexes for visual information such as color and texture, semantic indexes for non-quantifiable abstract information, and keyword indexes for more abstract information. The methodologies to integrate the query results from the separate searches through different types of indexes are investigated. To demonstrate the effectiveness of the retrieval mechanism, a prototype content-based image retrieval system has been designed and implemented.
\end{abstract}

\section{Keywords}

Image database, content-based retrieval, complex similarity query, 


\section{INTRODUCTION}

Many research groups are developing the multimedia information system that will allow users to retrieve images based on their contents. The contents to describe the image are extracted automatically from the image by image processing subsystem or annotated manually by human intermediaries. The selection of contents influences largely on the effectiveness of the system and it usually depends on the application domain. However, it is general that the more there is information describing the image content, the better the image retrieval system can discriminate the desired ones among the images in a database.

To facilitate the fast search based on the various types of information the indexes are needed, and we use separate indexes for the different feature sets describing the image content, because the feature sets have their own intrinsic characteristics which cannot be mixed together. The visual information such as color and texture is quantifiable and can be ordered in a measurable domain space. On the other hand, the non-quantifiable abstract information such as subject cannot be measured and thus cannot be ordered.

In such an environment with multiple feature sets, to develop the methodologies for processing of queries with multiple predicates including more than one similarity predicate, which are called complex similarity queries, is necessary. However, there are some difficulties of processing the complex similarity queries because the types of query results from the processing of the individual predicates are different. For example, the type of query result from one predicate is a set of values, while the type of query result from other predicate is an ordered list.

This paper will present the indexing scheme and the query processing methodologies for complex similarity queries. The rest of the paper is organized as follows. In section 2, we provide the data model for description of the image content. The index structures for the identified image contents and the processing methods for complex similarity queries are provided in section 3. Some experiments on the complex similarity queries are performed in section 4 . The conclusions are made in final section.

\section{IMAGE DESCRIPTION}

During database population (i.e., the process of creating a database) images are processed to extract features describing their content, and the extracted features are stored as metadata together with raw image in an image database. Queries are specified using the metadata. In a query, features from the database are compared to corresponding features from the query specification to determine which images are a good match.

We describe the content of an image using three set of attributes: 1) a set of visual features, 2) a set of semantic features, and 3) a set of keywords. The visual 
features would be colors, textures, shapes, positions, and so on. The semantic features include non-quantifiable or abstract information that should be extracted manually by the interpretation of human intermediaries. For example we can enumerate the following semantic features of an image:

- type: painting, scenery, portrait, and so on,

- subject: mountain, sea, animal, flower, architecture, and so on,

- title: title of image,

- perspective: aerial, ground, or close-up,

- orientation: horizontal or vertical,

- date: date when the picture is shot.

Keywords are words or sequence of words which describe the characteristics of the image that can not be represented with simple common attributes. Keywords contain the highest level of image abstraction among other features.

\section{INDEXING AND QUERY PROCESSING}

This section describes the indexing and query processing scheme to deal with the complex similarity queries. For a small database, sequential scanning of the features followed by straightforward similarity computations is adequate. But as the database grows, this procedure can be too slow, and indexes should be built to speed up the queries. Our indexing scheme consists of three types of indexes which correspond to three types features identified, respectively. The access to the image objects is performed only through these indexes and the query processing subsystem should use these indexes.

\subsection{Visual Indexes}

We assume that a set of $n$ visual features have been extracted automatically or manually from each image. They may be dominant colors, textures, and shapes, and so on. However, the only visual features currently used in our system are color and texture. When we represent a set of $n$ visual features as an $n$-sized tuple, $A_{v}=\left(f_{1}, f_{2}, \ldots, f_{n}\right)$, it can be mapped to a point in an $n$-dimensional visual feature space. We use the HG-tree (Cha, 1996) (Cha, 1998) as our underlying index structure for organizing the visual feature based indexes. The HG-tree is a multidimensional point index structure to index point data in multidimensional domain space. We select the HG-tree because it outperforms most of other multidimensional point index structures in a wide range of query performance comparisons and the $n$-sized feature vector is naturally represented as a point in an $n$-dimensional domain space. The HG-tree guarantees the storage utilization of $66.7 \%$ in worst case, and it has typically achieved more than $80 \%$. Moreover, it is fairly robust with respect to high dimensionalities, and it achieves good response time (Cha, 1998). 
Using the HG-tree, all $n$-dimensional visual feature values are transformed into 1-dimensional points using space-filling curve, and specifically Hilbert curve (Hilbert, 1891), before they can be used. A space-filling curve is a mapping that maps the unit interval onto the $n$-dimensional unit hyper-cube continuously. The desirable features of the Hilbert curve are that the points close on the Hilbert curve are close in the domain space, and the points close in the domain space are likely to be close on the Hilbert curve.

In the HG-tree, since all data points are represented by locations on the 1dimensional Hilbert curve there is no need to consider the problem caused by $n$ dimensional domain space. This promotes the deferred node splitting (Comer, 1979) to be used when node overflow occurs, and therefore guarantees the worst case storage utilization of $66.7 \%(2 / 3)$. To minimize the dead space (i.e., the space which does not include any actual data but covered by the directory region) of the node, the HG-tree encloses a set of entries in a node by minimum bounding interval (MBI). MBI is the smallest interval on the Hilbert curve, which completely encloses all the directory regions or data points at lower level.

The HG-tree consists of internal and leaf nodes. A leaf node contains at most $C_{l}$ entries of the form

(oid, $H)$

where $C_{l}$ is the capacity of the leaf node, oid is a pointer to the object in the database, and $H$ is the Hilbert value for $n$-dimensional visual feature vector. An internal node contains at most $C_{n}$ entries of the form

(ptr, I)

where $C_{n}$ is the capacity of an internal node, $p t r$ is a pointer to the child node, and $I$ is the MBI. The MBI is represented by two Hilbert values at both ends of the interval:

$$
I=\left(H_{1}, H_{2}\right) \text {, }
$$

where $H_{1}$ is the starting point and $H_{2}$ is the ending point on the bounding Hilbert curve. We maintain these entries in Hilbert order.

\subsection{Semantic Indexes}

A fixed-set of tuple to describe the semantic features of an image is represented by $A_{s}=\left(s_{1}, s_{2}, \ldots, s_{k}\right)$ such that $s_{i} \in D_{i}$ for $1 \leq i \leq k$. Where $D_{i}$ for $1 \leq i \leq k$ is a domain space of the $i$ th attribute $s_{i}$. The tuple $A_{s}$ is said to be a relation in relational data model. We have $k$ hash functions $H_{i}: D_{i} \rightarrow W_{i}$, for $1 \leq i \leq k$, where $W_{i}=\left\{0,1,2, \ldots, 2^{i \max }-1\right\}$, and $2^{i \max }-1$ is the maximum allowable hashed value. We construct the semantic indexes with this hashed tuple $H_{s}=\left(H_{1}\left(s_{1}\right), H_{2}\left(s_{2}\right), \ldots\right.$, $\left.H_{k}\left(s_{k}\right)\right)$. Duplicate hashed tuple may be introduced due to collisions. If a data page is filled up by duplicate tuple, multiple data pages can be chained. However, such hash collisions for all organizing attributes will not be frequent.

We employ the HG-tree as our underlying index structure for organizing semantic indexes unlike other image/video retrieval systems such as QBIC 
(Flickner, 1995) and Chabot (Ogle, 1995) which use B-tree (Comer, 1979) as their index structure to index semantic or tabular data. The B-tree is a primary indexing scheme, and thus indexes are constructed on the primary attribute. If it is required to provide a fast access on other attributes, another B-trees constructed on that attributes are also needed. Since we regard the $k$-sized semantic tuple $A_{s}$ as a set without some specific primary keys, we construct a single multi-attribute (SMA) index based on the HG-tree instead of constructing single B-tree index based on a primary key or constructing multiple single-attribute (MSA) B-tree indexes.

There are some important advantages of using SMA index as compared to MSA. First, the clustering of index pages and data pages on disk can drastically reduce the number of $\mathrm{I} / \mathrm{O}$ operations needed for database accesses. Second, when new records are inserted into or deleted from a database, SMA index organization needs only single update for its index. MSA index, in contrast, require multiple updates since the number of indexes is multiple. Therefore, maintaining the consistency of indexes in SMA index organization is simpler than that in MSA index organization.

\subsection{Keyword Indexes}

The signature file has proved to be a convenient indexing technique for text and multiattribute retrieval (Christodoulakis, 1984) (Deppisch, 1986) (Lee, 1989), (Lin, 1992), (Roberts, 1979), (Zezula, 1991). Multidimensional index structures such as K-D-B tree (Robinson, 1981), grid file (Nievergelt, 1984), R-tree (Guttman, 1984), $\mathrm{R}^{*}$-tree (Beckmann, 1990) are not appropriate for indexing text data represented by keywords. Because they assume the dimensionality of the domain space, which is the number of keywords in the case of keyword, is small and constant. The number of keywords given by users to query image databases is variable. Moreover, most of the multidimensional space index structures suffer from the dimensionality curse which say that as the dimensionality increases, the efficiency of the search drastically decreases. Therefore, we chose the signature file technique as our indexing method for keywords.

A lot of research has been done on the improvement of the performance of a signature file. However, most of the researches have been performed for static environments where update operations are rarely occurred. Our index design scheme requires a dynamic data environment, which means that the signature file must be allowed to grow and shrink. The two representative dynamic signature files are S-tree (Deppisch, 1986) and Quick,filter (Zezula, 1991). The main idea of the S-tree is to group adjacent signatures in pages and build a B-tree on top of them to provide direct access to the leaf signature pages. However, the major problem of the S-tree is that the performance is degenerated as the query signature weight becomes lower. The number of 1 's in a signature is called the signature weight. In the Quick filter, a signature file is partitioned by a hash function and the partitions are organized by linear hashing. Therefore, it is 
appropriate for the dynamic environment where updates are occurred frequently and results in good performance in the queries with high signature weights. However, when the distribution of signatures is nonuniform, then similar signatures are frequently generated and therefore the overflow rate increases and the storage utilization decreases.

To attack these performance disadvantages of existing dynamic signature files, we combine the concepts of the frame-sliced signature method (Lin, 1992) and the HG-tree. At first, a signature is divided into $s$ frames, and $c$ frames are selected out of a total of $s$ frames using one hash function $h_{1}$. To make up the word signature (i.e., the signature corresponding one keyword) $m$ bits are set to " 1 " in the selected $c$ frames using the second hash function $h_{2}$. The frame signature is constructed by superimposing the parts belong to the corresponding frame of word signatures. At last, the image signature representing the description of an image is constructed by concatenating the frame signatures.

The leaf and internal node structures of the HG-tree for keyword signatures are slightly modified to accommodate the keyword indexes. The entry in a leaf node has the form (oid, $F$ ), where oid is a pointer to the raw image in the database and $F$ is an image signature which consists of $s$ frames $\left(F_{1}, F_{2}, \ldots, F_{s}\right)$. The entry in an internal node has the form (ptr, $S$ ), where $p t r$ is a pointer to the child node and $S$ is a signature made by superimposing the image signatures of the child nodes.

\subsection{Processing Complex Similarity Queries}

Figure 1 shows the procedure for processing the complex similarity query. The predicates of the query are interpreted to appropriate feature values, which are visual feature values, semantic feature values, or keywords, during query processing. These feature values are evaluated and searched through corresponding indexes. Finally, Object IDentifiers, OIDs, are acquired and resolved to answer a complex similarity query.

The result of a $k$-nearest neighbor query (or similarity query) based on the visual feature values is a sorted list with $k$ most similar images. On the other hand, the results of queries based on the semantic features and keywords are almost sets of images which satisfy the queries. When the complex similarity queries are issued to retrieve more exact matches, the search procedure must synthesize the results of different types of queries.

Example 1. Let us consider the query $Q_{1}$ where a user wants to retrieve 5 most similar image to a given image $I$ and whose subject is 'animal':

$\mathrm{Q}_{1}$ : Find 5 images most similar to the image $I$ with subject $=$ 'animal'.

In this case, the query result is probably a list with 5 objects sorted by the visual similarity to the image $I$, where the value of subject attribute is animal. 


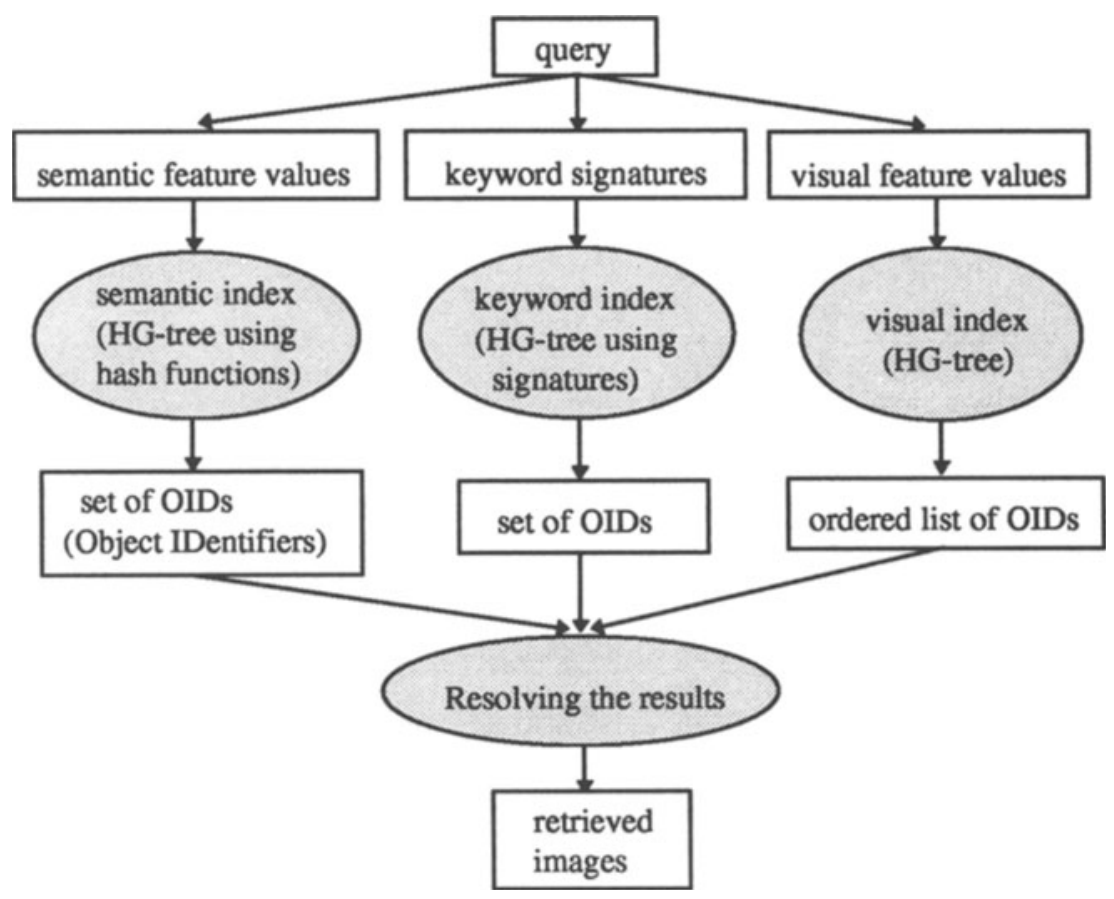

Figure 1 Processing Complex Similarity Queries

A reasonable way to evaluate this type of queries, in which the result of one query (or predicate) is a set and the result of the other query is a sorted list, would be to first evaluate the query whose result becomes a set, and then to find the sorted list consisting of the required number of objects from the set. Therefore, to evaluate the query $Q_{1}$ in Example 1, we determine all objects that satisfy the predicate subject $=$ 'animal', and then obtain similarity scores of the objects, and finally return 5 objects which have highest similarity scores.

Example 2. Let us now consider the query $\mathrm{Q}_{2}$ with two similarity predicates where a user wants to retrieve 10 best matches which are similar to both of images $I_{1}$ and $I_{2}$ :

$\mathrm{Q}_{2}$ : Find 10 images most similar to both images $I_{1}$ and $I_{2}$.

There are two ways to deal with this kind of complex similarity queries. One way is to transform the query with multiple query images to the query with only one predicate containing an image which is best matched to all query images. In the query $\mathrm{Q}_{2}$, since the images $I_{1}$ and $I_{2}$ can be mapped into two points $P_{1}$ and $P_{2}$, respectively, in the same $n$-dimensional feature space, we can find a point $P$ which is closest to both points $P_{1}$ and $P_{2}$. Then we retrieve 10 objects closest to the single point $P$. 
The other way is to find two result sets S1 and S2 which have at least 10 most similar images in common. Each result set is a set of pairs (oid, s) where oid is an object identifier of image and $s$ is its similarity value. Then, the overall similarity of the objects in both sets $\mathrm{S} 1$ and $\mathrm{S} 2$ can be computed as follows:

$$
\text { overall similarity of object } x=\prod_{i=1}^{m} s_{i}(x)
$$

where $s_{i}(x)$ is the similarity value in range $[0,1]$ of an object $x$ in the result set $S_{i}$, $x$ is the object which is included in all result sets, and $m$ is the number of result sets, i.e., the number of similarity predicates. The similarity value of 1 denotes the exact match and the 0 shows the maximum difference. This similarity function employ the probability function on the independent predicates as the combining rule for independent similarity scores.

It should be noted that the query results from the above two methods may be different. In the former method, the search target is changed to the object most common to the given query objects, on the other hand, in the latter method, the most similar objects among the separately selected best matches are chosen. This difference for best matches may make the final results different. To determine which method produces more exact results is not trivial and can be dependent on the user's viewpoint. One obvious fact is that the former is far more efficient than the latter. Because there is only one search to the index in the former and on the other hand, there are many index searches as the number of conjuncts in the latter.

\section{4}

\section{EXPERIMENTS}

To test the effectiveness of our indexing and query processing mechanism for content-based image retrieval, we have constructed an image database that has a 1,064 images. The images are 256-color bitmaps with a variety of characteristics. In the database, images are stored together with three kinds of attributes, i.e., semantic features, visual features, and keywords, so that images can be retrieved from these descriptions. The visual features are extracted automatically by image parsing subsystem and the semantic features and keywords are inserted manually in database population time.

\subsection{Visual Feature Extraction}

To acquire the visual features that characterize images we used statistical color moments of the histogram of the image because color has excellent discrimination power in image retrieval system. Since most histogram bins of an image are sparsely populated and only a small number of bins have the majority of pixel counts, we used only the largest 32 bins (in terms of pixel counts) as the representative bins of the histogram. We used first two moments of the histogram as descriptors of an image: 


$$
\mu_{i}=\frac{1}{n} \sum_{j=1}^{k} f_{i j} x_{i j}, \sigma_{i}=\left(\frac{1}{n} \sum_{j=1}^{k} f_{i j}\left(x_{i j}-\mu_{i}\right)^{2}\right)^{\frac{1}{2}}, i=1,2,3
$$

where $x_{i j}$ is the value of color component of the $j$ th bin, $f_{i j}$ is the frequency of $x_{i j}, k$ is the number of total bins, i.e. 32 , and $n$ is the total number of pixels in the histogram. Since we used the RGB color model, the $i$ th color component corresponds to one of red, green, and blue. The first moment, $\mu_{i}$, defines the average intensity of each color component. The second moment, $\sigma_{i}$, is a measure of contrast that can be used to establish descriptors of relative smoothness.

Measures of global color statistics using only histograms suffer from the limitation that they carry no information regarding the relative position of pixels. To overcome this limitation to some extent, we divided the image into 4 subareas and computed 2 moments for each sub-area, resulting in a 24 (=2 moments $\times 3$ color components $\times 4$ sub-areas) features for an image.

Using this 24-dimensional feature vector, we estimate the similarity, $s(S, T)$, between two color histograms $S$ and $T$ as follows:

$$
s(S, T)=1-\frac{\sum_{k=1}^{4}\left(\sum_{i=1}^{3}\left(\left|\mu_{i k}(S)-\mu_{i k}(T)\right|+\left|\sigma_{i k}(S)-\sigma_{i k}(T)\right|\right)\right)}{\Delta},
$$

where $\Delta$ is a normalizing factor.

\subsection{Sample Similarity Queries}

Fig. 2 shows the results of two sample 12-nearest neighbor queries:

(a) Query 1:Find 12 images most similar to a given image I;

(b) Query 2: Find 12 images most similar to a given image $I$ whose subject $=$ 'animal'.

The image on the upper-left corner in Figure 2(a) and 2(b) is a given query image $I$ and 12 most similar images are retrieved in left-right and top-down sequence: In Figure 2(a), tigera4.bmp is the query image and, of course, it is the most similar image, bench1.bmp is the second similar image, and detail14.bmp is the 12nd similar image. The result of Query 1, i.e., Figure 2(a), was obtained only using color and texture visual features. Obviously, all images retrieved from a real image database have similar color properties to the given query image. On the other hand, the semantic feature, subject = 'animal', are used together with the visual features in the processing of Query 2 and whose result is shown in Figure 2(b). Obviously, it is shown that the more the features are specified in the query, the higher the selectivity, i.e., the ratio of the expected number of answers over the total number of data in the database, is increased. However, the more the features are specified in the query, the higher the search cost increases. 


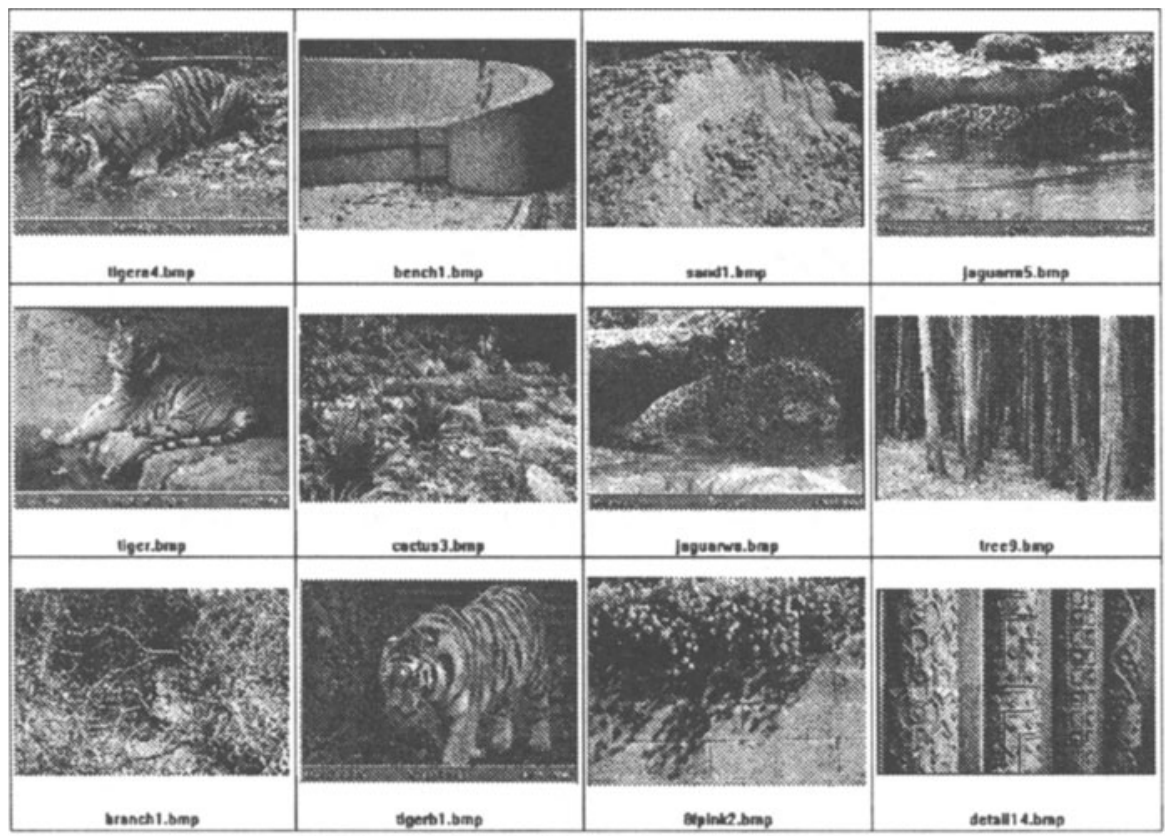

(a) Query result using only visual features

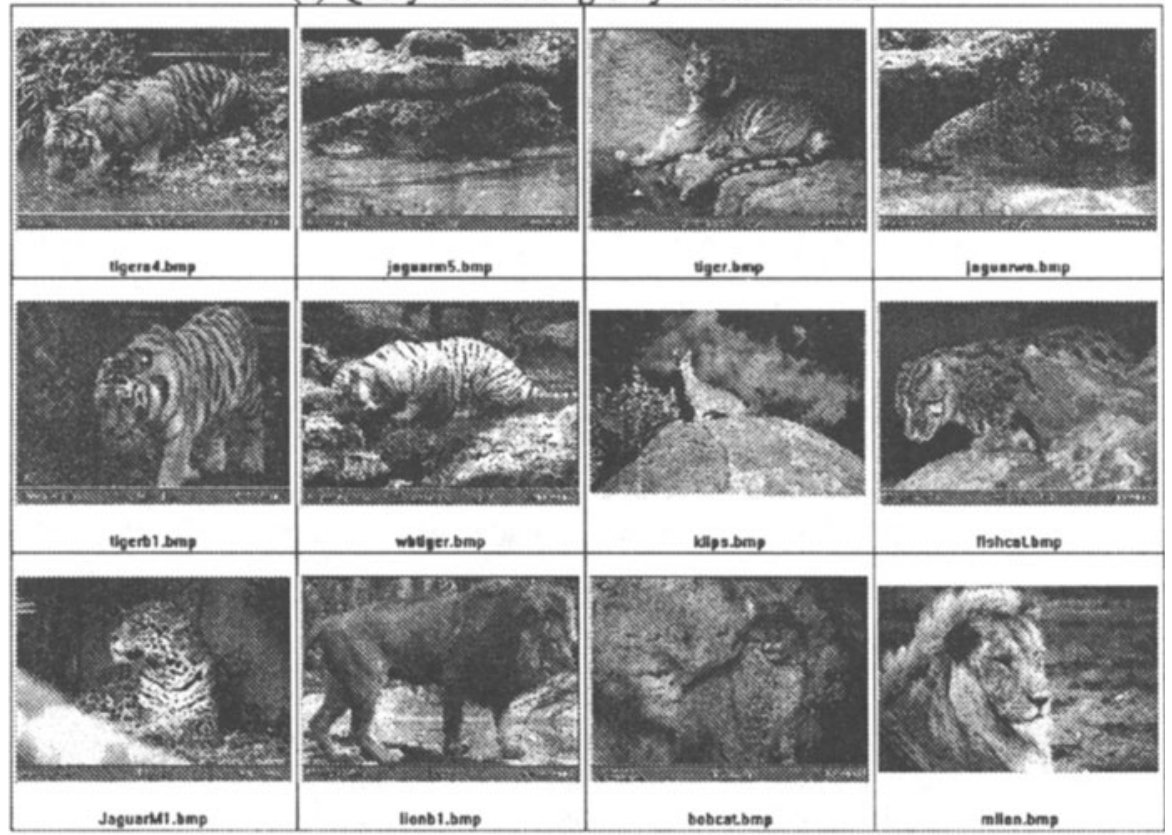

(b) Query results using visual features and semantic feature

Figure 2. Two sample 12-nearest neighbor queries 


\section{CONCLUSIONS}

In this paper, we present the indexing and processing mechanism for complex similarity queries in content-based image retrieval system. We model an image object with three kinds of sets which have visual, semantic, and keyword attributes. Corresponding to three sets of image features, we construct three types of indexes separately, because each feature set cannot be mixed together in nature. The techniques for processing the complex similarity queries are also provided. It resolves the inconsistency of the result sets from different types of query predicates.

There are some important issues not discussed thoroughly in this paper. First, what kind of data model is most appropriate to describe the image content, though we represent the image content with unstructured flat form. Probably, object-oriented approach can be an candidate to organize the image content. Second, we did not consider in depth the performance characteristics of the processing techniques for complex similarity queries. Our future work will deal with these problems.

\section{REFERENCES}

Beckmann, N., Kriegel, H.-P., Schneider, R., and Seeger, B. (1990) The R*-tree: An efficient and robust access method for points and rectangles. Proceedings of ACM SIGMOD International Conference on Management of Data, 322331.

Cha, G.-H. and Chung, C.-W. (1996) HG-tree: An Index Structure for Multimedia Databases. Proceedings of the 3rd International Conference on Multimedia Computing and Systems, 499-452.

Cha, G.-H. and Chung, C.-W. (1998) A New Indexing Scheme for Content-Based Image Retrieval. Multimedia Tools and Applications, 6(3).

Comer, D. (1979) The Ubiquitous B-tree. ACM Computing Surveys, 11(2), 121137.

Christodoulakis. S. and Faloutsos, C. (1984) Signature files: An access method for documents and its analytical performance evaluation. ACM Transactions on Office Information Systems, 2(4), 267-288.

Deppisch, U. (1986) S-Tree: A Dynamic Balanced Signature Index for Office Retrieval. Proceedings of the ACM Conferences on Research and Development in Information Retrieval, 77-87.

Flickner, M. et al. (1995) Query by Image and Video Content: The QBIC System. IEEE Computer, 23-32.

Guttman, A. (1984) R-Trees: A Dynamic Index Structure for Spatial Searching. Proceedings of the ACM SIGMOD International Conference on Management of Data, 47-57. 
Hilbert, D. (1891) Uber die stetige Abbildung einer Linie auf ein Flachenstuck. Math. Annalen, 38.

Lee, D.L. and Leng, C.-W. (1989) Partitioned Signature Files: Design Issues and Performance Evaluation. ACM Transactions on Office Information Systems, $7(2), 158-180$.

Lin, Z. and Faloutsos, C. (1992) Frame-Sliced Signature Files. IEEE Transactions on Knowledge and Data Engineering, 4(3), 281-289.

Nievergelt, J., Hinterberger, H., and Sevcik, K.C. (1984) The grid file: an adaptable, symmetric multikey file structure. ACM Transactions on Database Systems, 9(1), 38-71.

Ogle, V.E. and Stonebraker, M. (1995) Chabot: Retrieval from a Relational Database of Images. IEEE Computer, 28(9), 40-48.

Roberts, C.S. (1979) Partial-Match Retrieval via the Method of Superimposed Codes. Proceedings of the IEEE, 67(12), 1624-1642.

Robinson, J.T. (1981) The K-D-B-Tree: A Search Structure for Large Multidimensional Dynamic Indexes. Proceedings of the ACM SIGMOD International Conference on Management of Data, 10-18.

Sacks-Davis, R., Kent, A., and Ramamohanarao, K. (1987) Multikey Access Methods Based on Superimposed Coding Techniques. ACM Transactions on Database Systems, 12(4), 655-696.

Zezula, P., Rabitti, F., and Tiberio, P. (1991) Dynamic Partitioning of Signature Files. ACM Transactions of Information Systems, 9(4), 336-369.

Guang-Ho Cha received his Ph.D. in 1997 from Korea Advanced Institute of Science and Technology. He is currently a full-time lecturer in the Department of Multimedia Engineering at Tongmyong University of Information Technology. From 1989-1996 he worked on the development of a videotex system and an internet service system in DACOM.

Chin-Wan Chung is an associate professor in the Department of Computer Science at Korea Advanced Institute of Science and Technology since 1993. Prior to that, he was a staff research scientist in the Computer Science Department at General Motors Research Lab. His current research interests include objectoriented databases, geographic information systems, and multimedia databases. 